\title{
La visión sobre el cuerpo desde de las tradiciones del lejano Oriente
}

The view from on the body of the traditions of the far East

\section{José Arlés Gómez Arévalo}

Doctor en Teología, director de la línea de investigación en Pedagogía del Doctorado en Educación de la Universidad Santo Tomás de Aquino (USTA), Bogotá - Colombia, e-mail: angel777abc@hotmail.com

\section{Resumen}

El presente artículo hace parte del trabajo investigativo desarrollado por el Grupo CienciaEspiritualidad que indaga por las posibles conexiones que se establecen entre algunas epistemologías de Occidente y las tradiciones del lejano Oriente; dicha investigación, en su tercera fase, indaga por las relaciones hombre-naturaleza. En esta etapa se reflexiona por el papel del cuerpo en las tradiciones taoísta, budista e hindú quienes desde sus prácticas socio-culturales, filosóficas y espirituales, fundamentan una mirada del cuerpo desde su integración unitaria con la mente y el espíritu. Entender esta conexión constituye un valioso aporte a las actuales reflexiones que sobre el ser humano como ser integral se realizan en algunas epistemologías emergentes.

Palabras Clave: Cuerpo. Mente. Espíritu. Tradición. Unidad. 


\section{Abstract}

This article is part of the research work carried out by the Science-Spirituality Group that explores the possible connections established between some Western epistemologies and traditions of the Far East, such research, in its third phase, explores the relations man-nature. This stage reflects the role of the body in Taoist traditions, Buddhist and Hindu practices who from their socio-cultural, philosophical and spiritual foundation of a body look from unitary integration with mind and spirit. Understanding this connection is a valuable contribution to the current reflections on the human being as a whole is made in some emerging epistemologies.

Keywords: Body. Mind. Spirit. Tradition. Unity.

\section{Introducción}

"Las culturas constituidas dan al cuerpo una preponderancia mayúscula en su ideología. Los cristianos los entierran. Los vikingos los quemaban.

Los egipcios los preparaban para que duraran varios siglos. En el lejano Oriente, no se podía concebir el viaje a través de este mundo para llegar a trascender, sin el cuerpo en unidad con la mente y el espíritu." (Wuatanabe)

Antes de iniciar este importante tema de la concepción del cuerpo en el lejano Oriente, es importante recordar que de acuerdo con el historiador y pensador René Guénon ${ }^{1}$ (1886-1951), crítico vigoroso del Occidente moderno, es posible hablar de una mentalidad oriental opuesta en su conjunto

1 Según Guenon (1964, p. 53), tendríamos así una civilización occidental y varias orientales. Sin embargo, la unidad cultural de la civilización occidental moderna asentaría en un conjunto de tendencias que constituyen una cierta conformidad mental, una simple unidad de hecho, sin principio, desde que el Occidente se apartó de la Cristiandad, su principio constitutivo hasta la Edad Media. mientras las civilizaciones orientales, por más diversas que sean, cada cual asentando sobre un principio de unidad distinto, traen todas ciertos rasgos culturales comunes, principalmente cuanto a los modos de pensar, lo que permite decir que existe, de un modo general, una mentalidad específicamente oriental. 
a la mentalidad occidental, pero no se puede hablar de una civilización oriental como se habla de una civilización occidental, ya que hay varias civilizaciones orientales claramente distintas (GUÉNON, 1964).

El punto de partida para caracterizar la concepción del cuerpo en el lejano Oriente revela un profundo contenido cultural, espiritual y ético ligado a sus tradiciones espirituales. En este sentido, y en primera instancia, podemos decir que el lejano Oriente es fundamentalmente China, India y Japón, no hace falta subrayar el inmenso aporte cultural, filosófico y científico de estas grandes tradiciones orientales.

Por otro lado, la recuperación de la unidad originaría de cuerpomente-espíritu, ayuda a superar la separación entre ciencia, filosofía y espiritualidad: esta unidad además es clave para el diálogo intercultural, ya que tanto en Oriente como en Occidente ${ }^{2}$, se halla la búsqueda hacia el origen del ser mediante la actividad no sólo intelectual sino contemplativa, de lo que encontramos testimonios a lo largo de la historia del pensamiento oriental (MASIÁ CLAVEL, 1996).

\section{Concepción de cuerpo en el lejano Oriente}

Es un punto bien establecido por los sabios y pensadores, además de los historiadores tanto orientales como occidentales, se puede afirmar que la concepción del cuerpo en el lejano oriente está íntimamente ligada a la concepción unidad del mismo con la mente y el espíritu; un ejemplo de ello es que la liberación no sólo es posible en este cuerpo y en la tierra, sino sólo a través de "este cuerpo" y en la tierra esto significa que es posible la liberación o mosha, como lo afirma la tradición hindú, porque el hombre ha trascendido a través de su propio cuerpo (GÓMEZ, 2006).

2 García Bacca (1987, p. 74) distingue dos planos: El hombre como tema (El cuerpo como algo definido, estable y permanente) Y El hombre como problema (El cuerpo como evolución, dinamismo y progreso). Esta distinción entre lo permanente y lo transeúnte es una de las interpretaciones de la realidad del hombre que divide las escuelas filosóficas desde la antigüedad. Dentro de estas realidades se incluye la realidad corporal que da pie a planteamientos tanto filosóficos, culturales como espirituales. 
De la misma manera, es importante decir que la visión de la corporeidad $^{3}$ en la tradición oriental, está fundada en la experiencia mística y espiritual de la meditación; así mismo, es importante aclarar que la corporalidad incluye, en las tradiciones del lejano Oriente, también la espiritualidad. Es decir, el punto de partida del pensamiento oriental es la armonía biopsicoespiritual del hombre con su entorno. Los métodos de meditación tienen un valor y un efecto muy importante para contemplar qué es el ser humano, no solamente en un sentido material sino también espiritual, psicológico, médico y gimnástico. Por tanto, comenta el filósofo japonés Yasuo Yuasa, en el mundo actual habría que recuperar un auténtico sentido de la sabiduría de esas tradiciones arcaicas que fueron rechazadas por la razón moderna occidental (YUASA, 2001).

\section{El sentido del cuerpo en la Tradición Hindú}

Si tomamos la teoría de los "Pancha-Kosas" o cinco capas, de la cultura hindú, se piensa que el ser humano está constituido por una sucesión de envoltorios, a la manera de capas de cebolla. El cuerpo (físico), así como la mente (emocional) y el intelecto son tres de estos estratos. Los otros dos son la energía (que en la tradición hindú equivale al prana) y el espíritu.

En la misma tradición hindú, se mencionan así mismo diferentes "cuerpos": el "burdo", el "sutil" y el "causal", donde -aproximadamenteel primero sería el cuerpo físico, el segundo el conglomerado emoción/ intelecto y el tercero lo que podríamos traducir como "espíritu", llamado "causal" porque es la causa de los otros dos.

De la misma manera que en la tradición hindú, encontramos que en la milenaria China con sus tradición taoísta y posteriormente con la influencia del budismo en sus tres formas o ramas, todo es conciencia,

3 Se puede definir corporeidad como "la vivenciación del hacer, sentir, pensar y querer" (ZUBIRI, 1986). La corporeidad se refiere igualmente al ser humano, y por tanto, el ser humano es y vive sólo a través de su corporeidad. 
todo es energía; la así llamada energía Chi o ki que equivale al prana ${ }^{4}$ de los hindúes, indica también el cuerpo físico, lugar donde se manifiesta la enfermedad o la salud que se origina en el campo energético.

Según el hinduismo, el budismo y el taoísmo, todo cuerpo vivo está rodeado por un patrón holográfico de energía, que aporta información para el desarrollo de los seres humanos, desde el estado embrionario hasta su vejez. Este patrón holográfico, que posteriormente fue comprobada por la ciencia occidental mediante la visualización utilizada por la fotografía electrográfica Kirlian ${ }^{5}$, para cuerpos de alta frecuencia, alto voltaje y bajo amperaje, mostrando emanaciones brillantes, o "Aura", o "campo bioenergético" o "campo electromagnético luminoso". En la misma tradición hindú, en el cuerpo humano existen numerosos puntos vitales llamados chakras ${ }^{6}$ situados entre el cuerpo etéreo y el llamado cuerpo burdo. Son, por tanto, "puertas" hacia los distintos cuerpo sutiles. Al ser despertados alcanzamos ciertos poderes físicos, felicidad y finalmente el "despertar la consciencia" (OSHO, 2012).

4 En sánscrito, prāna por lo que se refiere al principio vital o el poder de la energía vital así como rūah en el hebraico, psyché o pneuma en el griego. En el pensamiento cristiano, podemos encontrar el espíritu, que es considerado como el principio vital del ser humano. En la China antigua, se relacionaba la noción del chi con los fenómenos naturales (lluvia, nubes y viento) que aportan mucho a la formación de la vida de todos los vivientes: Ios fenómenos naturales, a veces, pueden causar desastres en la tierra, pero sobre todo aportan un don, que hace vivir a cuanto vive en la tierra. Originalmente, la noción del chi se identifica sobre todo con el fenómeno del viento, que trae el cambio del tiempo e influye en el cultivo de los granos. A partir de ahí se ha desarrollado la noción del chi, relacionándolo con la respiración humana. El acto de respirar es lo principal para las personas vivientes. La actividad de la respiración humana, según el pensamiento antiguo chino, está relacionada con la tierra y el cielo y la corriente del viento.

5 Fue inventada por el matrimonio que la da su nombre, Davidovich y Valentina Kirlian, en el año 1939. En el laboratorio del Hospital de Alma-ata en la Unión Soviética, este matrimonio experimentaba con campos magnéticos de alto voltaje. La cámara Kirlian permite fotografiar el efecto corona que se genera con cualquier objeto (vivo o inanimado). Los cientificos opinan que el estudio de la intensidad de este fluido, permite analizar el estado anímico y físico de una persona. Mediante la cámara afirman que se obtiene lo que se ha denominado el efecto kirlian o halo luminoso, es decir, una especie de aureola energética que rodea al objeto fotografiado.

6 La palabra sánscrita chakra significa 'círculo' o 'disco'. Proviene posiblemente de la raíz sánscrita char. 'moverse [las personas, los animales, el agua, los barcos, los astros]'; o de la raíz sánscrita kri (en el nirukta, 6.1.12). A su vez el término chakram deriva del indoeuropeo, de la que derivaron: el griego kiklós y esta palabra griega generó la palabra española ciclo. 
Los vórtices o chakras están unidos a través de una serie de canales denominados nadis; éstos y los meridianos de acupuntura china son lo mismo. Pero existen más nadis que meridianos, en total, unos 72.000. La función principal de los chakras es absorber la energía universal y metabolizarla alimentando los distintos cuerpos del ser humano para finalmente irradiar energía hacia el exterior desde el interior hasta que lo de dentro y lo de fuera se fusionen. Es la llamada "omniscencia de la consciencia". Dentro de este complejo sistema energético existen siete chakras básicos o principales que se distribuyen desde la parte más baja de la columna vertebral donde se encontraría la kundalini, hasta la parte más alta de la cabeza. Los cuales se describen a continuación:

- Muladhara que es la puerta al cuerpo físico tanto masculino como femenino; las zonas vitalizadas de este chakra son: Glándulas suprarrenales, vértebras sacras, riñones, vejiga, y parte final del intestino. Es el vórtice encargado de los mecanismos de supervivencia que mantienen el cuerpo físico vivo: dinero, sexo, alimento y cobijo. Contiene, en forma de energía-raíz, bija, las huellas kármicas de la familia e información social y familiar que conforman la idea de realidad que tiene una persona.

- Svadhistana: cuyo significado es dulzura y morada del Ser. Su localización está a la altura de la próstata o del punto $\mathrm{G}$ de las mujeres. Cerca de las vértebras sacras, además de los testículos, ovarios, nervio ciático y vértebras dorsales. Sus funciones es que a través de este vórtice percibimos las emociones de otras personas (clarisentencia): tiene que ver con los aspectos físicos de la existencia; también con la gente con la que nos relacionamos y con la claridad de nuestras relaciones y con la energía sexual, y es el punto a partir del cual enviamos y recibimos sensaciones sexuales.

- Manipura: Cuyo significado es "diamante puor". Su localización se encuentra a la altura del ombligo, cerca de las vértebras dorsales. Su función está relacionada con la voluntad y el poder. Sus Zonas vitalizadas son: Páncreas, hígado, bazo, estómago, duodeno, cólon, e intestino delgado. Es el punto de distribución en el cuerpo de las 
energías psíquicas. Vórtice de poder/control: voluntad, poder personal, autoridad, control de uno mismo y autoestima.

- Anahata: Su significado es "Intacto o sonido no causado". Su localización se encuentra a la altura del corazón cerca de las vértebras torácicas. Su función es: Amar y las zonas vitalizadas que compromete son el timo, el corazón, el aparato respiratorio y el nervio vago. Es el Chakra del amor, la afinidad, la compasión y el soporte: "Unidad" con la vida: amor divino/incondicional, perdón y apertura.

- Además, este chakra, conecta los tres centros inferiores fisico/emocionales con los tres vórtices superiores mental/espirituales. Es denominado a veces en las mujeres "corazón superior".

- Vishuda: Su significado es pureza. La localización se encuentra en la garganta. Su función es la comunicación, la inspiración, la expresión personal, de seguir nuestros sueños: vórtice a través del cual recibes la voz interior: clariaudiencia (audición clara). Guarda relación con la capacidad de recibir sustento, asumir responsabilidad de nuestras necesidades personales. Es el poder de la palabra hablada y del conocimiento superior. Cuando se activado ayuda a la creatividad, y a la conexión, así como a la capacidad de recibir y asimilar. Este centro es responsable del rejuvenecimiento y la longevidad; el hombre que lo domine alcanza la "inmortalidad" pues a partir de ese momento vive fuera del tiempo, más allá del pasado, presente y futuro los cuales domina. Con este chakra abierto comprendemos cuales son nuestros anhelos materiales y espirituales, así como entendemos que somos los responsables directos de cuanto recibimos y asimilamos, por lo tanto es sinónimo de integridad. El Yo Soy emerge y puede actuar en cualquier situación.

- Ajna: Su significado es percepción y orden. La localización es en la frente. Su función es la Intuición y el sentido unido al pensamiento. Las zonas vitalizadas son la glándula hipófisis, parte inferior del cerebro, ojo izquierdo. Es el chakra o vórtice de la clarividencia, intuición, sabiduría, visión personal: la visualización, imaginación y vislumbre. - Cuando se activa ayuda a la imaginación, fantasía, visualización. Dominio del espíritu sobre la materia. Percepción, 
conocimiento. Por él se puede entrar en el mundo de lo inmaterial, de lo aparentemente invisible para dotarnos de conocimiento.

- Sahasrara: Cuyo significado es el "Loto de mil pétalos". Su localización es la cima del cráneo. Su función es el conocimiento y el Sentido del todo y nada. Las zonas vitalizadas por medio de él son la glándula pineal, parte superior del cerebro y ojo derecho. Es el Chakra del conocimiento e intuición pura, viendo el propósito final de nuestras vidas: Libre albedrío/posesión del cuerpo. Las actitudes que acrecienta son la fe, los valores, la ética, el coraje y el humanitarismo. Cuando se activa concede felicidad, entendimiento, libertad, autorrealización. Es la luz del conocimiento y conciencia, es la visión global del universo, y amor y compasión infinitos. Y en algunos casos de desarrollo máximo se puede ver como un halo dorado de los hombres santos.

Con esta ilustración anterior, queda de manifiesto que el cuerpo, en este caso desde la tradición hindú con su rico aporte a la comprensión del ser humano, adquiere un matiz muchísimo más complejo que algunas miradas occidentales, ya que está fundamentado en la experiencia psicobioespiritual del hombre; es decir, el punto de partida del pensamiento oriental es la armonía psicosomática con la energía espiritual, de hecho los variados métodos de meditación tienen un valor y un efecto muy importante para contemplar qué es el cuerpo humano dentro del concepto mismo de hombre, no solamente en un sentido físico, sino también en un sentido psicológico, espiritual, gimnástico y energético.

Por último, en este apartado sobre el tema del sentido del cuerpo en consonancia con la mente y el espíritu, en la India milenaria, no se puede dejar de mencionar que uno de los grandes aportes es justamente el sistea de medicina natural denominado Ayurveda. En el idioma sánscrito Ayur significa vida y Veda conocimiento. Literalmente significa "la ciencia de la vida". El ayurveda, está concebido sólo como un sistema médico sino como un verdadero sistema de vida para promocionar la salud. Es probablemente el sistema curativo natural más antiguo del mundo ya que sus raíces proceden de la era Védica, hace más de 5.000 años. El Ayurveda es una medicina viva que trata tanto cuerpo como mente y espíritu. Por 
su concepción y su metodología se integra con la medicina moderna, complementando sus valiosos aportes y soluciones. Acepta y promueve la observación y la investigación científica, la cual ha permitido corroborar muchos de sus postulados y recomendaciones. Es un sistema efectivo en la prevención y la cura de enfermedades, la preservación de la salud y la promoción de la longevidad.

\section{La relación cuerpo-mente-espíritu a través de la respiración en el taoísmo en la antigua China}

La antigua China, aportó una de las tradiciones más importantes en el lejano Oriente: el taoísmo, esta se atribuye al sabio y filósofo Lao Tsé o Lao Tzi (siglo VI a.c). Se le atribuye haber escrito el libro del Tao Te Ching, obra esencial del taoísmo. De acuerdo con este libro, el Tao o el Camino, puede verse como el cambio permanente y éste es la verdad universal. Dentro de las dudas sobre su existencia y la etapa histórica en la que vivió, se cree que pudo ser contemporáneo del también pensador y filósofo chino Confucio.

En el taoísmo, se da mucha importancia a cultivar el ki a través de la respiración para cuidar la vida humana, tanto en el sentido de la salud física, como en el sentido de la propia mente. Según la teoría de Lao-Tsé, para cuidar la vida hay que concentrarse en respirar para hacerse transparente a la energía que circula por el propio cuerpo, y calmar el cuerpomente dejando los deseos que hacen turbarse a sí mismo. Cultivarse a sí mismo para poder mantener una buena condición de la salud cuidando esa energía vital, según Lao-Tsé, es el camino (Tao) de la virtud. Se dice así en la obra de Lao-Tsé que sostener el alma en sí misma, es importante para no perderse y mantener el equilibrio entre cuerpo-mente sin dejar de estar unidos.

Según la división aparente del Tao, Lao-Tsé hace hincapié en el hecho de que toda la naturaleza es relativa y se sucede de cambios constantes dialécticamente complementarios, llamándole así a los dos aspectos opuestos y complementarios llamados yin y yang, presentes en todo el devenir cósmico de la naturaleza, tanto en los orígenes metafísicos como 
en los seres vivos y el mundo fenoménico, aplicándose así también para analizar todo lo existente, incluidos los aspectos sociales y humanos que Lao-Tsé estudia en su filosofía.

El sabio chino, igualmente explica como el yin y el yang, no son elementos confrontados, sino que sus características opuestas se complementan para conformar así la Totalidad del Tao, siendo que de esa forma, las mutaciones naturales permiten que lo asociado como Yin se vuelva Yang, y viceversa, dando así el ritmo dialéctico que permite la fluidez natural de la Energía (Chi), la cual representa la vitalidad de lo existente, siendo de esa forma Yin y Yang la mecánica de funcionamiento dialéctico de la energía, en otras palabras, la relatividad y el cambio natural que permite la fluidez de la Naturaleza, la cual obtiene armonía al hallar equilibrio entre sus dos aspectos opuestamente complementarios.

La respiración es clave para inspirar el chi o ki del universo y hacerlo circular dentro del cuerpo y armonizarse con el Tao de la naturaleza ${ }^{7}$. La convivencia con la naturaleza, según Lao-Tsé, es lograr la mente pacífica como una corriente de agua que se transforma espontáneamente según la situación, pero el agua es un elemento imprescindible para la vida cotidiana. Por tanto, obtener la mente calma como una corriente natural del agua, se dice en la obra de Tao Te Ching, es el camino del cuidar la vida (Tao Te Ching, cap. 8, 66, 78). De la misma manera, habla Chuang-Tzu, cuidar el camino espiritual es calmar las pasiones y unirse con el camino de la naturaleza como agua pura que fluye en su forma natural (Los capítulos exteriores de Chuang Tsu, cap. 15).

De la misma manera, resulta interesante que en el texto del Tao Te King, la relación entre cuerpo-mente-espíritu, se evidencia de manera inmediata a través de la respiración que es representada como método

7 Sobre el origen del Tao, parece ser que Lao-Tsé plantea que al ser éste la esencialidad de todo, es entonces el Tao el origen de las cosas y no el Tao la creación de algo o alguien más, debido a que son las cosas definidas del universo las que fluyeron de las mutaciones constantes y consecuentes del Tao, por lo que éstas son las cosas que el hombre logra conocer y catalogar bajo nomenclaturas, pero el Tao en sí no es una cosa, sino que es la cosa en sí; no de la forma absolutista de imperatividad jerárquica, sino como esencia infinita generadora de los posteriores cambios que tomaron forma en las diferentes manifestaciones; por lo que tanto los seres vivos, los objetos inanimados, la Tierra misma y el Cielo, todos han de ser formas que surgieron de cambios anteriores de la propia naturaleza, siendo así la Naturaleza la Madre de todas las cosas.

Rev. Pistis Prax., Teol. Pastor., Curitiba, v. 6, n. 2, p. 651-669, maio/ago. 2014 
principal para el Tao de la virtud. La actitud de cultivar la energía del ki corpóreo-espiritual ordenando la respiración, se relaciona directamente con la salud, la virtud, política y también mística. Es decir, la contemplación del autocultivo para hacerse transparente a sí mismo es clave para cuidar la vida y también para el mundo exterior, la sociedad, la naturaleza. Esa espiritualidad se ha desarrollado a través de la historia china y se ha transmitido al Japón: la espiritualidad que acentúa la unión de lo corpóreo espiritual y el entrenamiento para recuperarla.

En el taoísmo, con relación al tema del cuerpo, es importante recordar que se destaca el concepto de wu-wei, "acción a través de la inacción del cuerpo" o también traducido como "acción sin esfuerzo", que no significa permanecer inmóvil el cuerpo sin hacer nada, sino evitar las intenciones explícitas y la voluntad que obstaculiza la fluidez armónica del mismo cuerpo con la naturaleza. Un ejemplo claro de la práctica corpóreoespiritual del wu-wei, lo constituyen el tai chi y el Qi gong o chi kung, ambas prácticas habituales en la actual China y que se han extendido por Occidente. El Tai Chi es lo que millones de chinos hacen cada mañana juntos en parques y grandes plazas. Es un antiguo arte marcial chino, con probados beneficios para la salud mental y física que se realiza mediante movimientos corporales relajados y armoniosos en combinación con la respiración y la concentración mental. Recomendado en diversas enfermedades y lesiones. Se basa en conceptos de la Medicina Tradicional China, y de la filosofía taoísta de la antigua hina como son la circulación del chi (ki o prana), los meridianos de acupuntura, la teoría del yin y el yang, la ley de los cinco elementos, etc.

El Tai Chi es una de las artes marciales internas, desarrolladas desde el monte del Wudang muy antigua tradición taoísta en China, por contraposición a las artes marciales externas cuyo mayor exponente está en Shaolin. Forma parte del cultivo corpóreo-espiritual taoísta ("xiulian"), el carácter meditativo que posee su ejecución, así como otras características avanzadas. El Taichi es una simplificación de su nombre: taijiquan (pronunciado "taichichuan").

De la misma manera, el Qi Gong, que se refiere al "aliento vital" o $\mathrm{Ki}$, a la energía que anima a todo el universo y en particular al hombre, Gong se refiere al "trabajo". Qi Gong o Chi Kung, significa el trabajo sobre 
la energía vital, o dicho en otras palabras: la gimnasia corporal-energética china que ayuda a restaurar, tonificar, vitalizar todo el cuerpo en armonía con el espíritu y la mente. El tai chi, es parte de la medicina tradicional china desde hace 2000 años, Los ejercicios de Chi Kung que se practican como complemento al Tai Chi Chuan están fundados en 3 principios:

- El control del movimiento, que debe ser natural, lento y tranquilo,

- El control de la respiración, que debe ser lento y sincronizado con los gestos,

- El control del pensamiento, que dirige el Chi, la respiración, el aliento al interior del cuerpo.

La práctica del Qi Gong o chi kung, procura una relajación del cuerpo, porque los movimientos son lentos, distendidos y ágiles. Esta relajación se profundiza con la respiración que disminuye. En ese estado, se siente el interior del cuerpo y se comunica al exterior, con la naturaleza.

\section{La perspectiva de la corporalidad "Shin-tai" en el marco de lo corpóreo-espiritual según el budismo zen japonés}

En este apartado, se han tomado los aportes de dos autores japoneses de capital importancia en el siglo XX: Tetsuro Watsuji (18891960) y su discípulo, Yasuo Yuasa (1925-2005). Ambos autores, estudiosos además de las tradiciones orientales de las corrientes de la filosofía occidental; por otro lado, pretenden recuperar la espiritualidad originaria en el ámbito del pensamiento oriental, tratando la cuestión del ser humano en su concepto de unidad corpórea-espiritual: Watsuji expone su captación del ser humano dentro del marco espacio-temporal de la relación y la originalidad de la convivencia humana con la naturaleza.

De entrada, es importante decir que el zen es fundamentalmente una práctica de auto-cultivo corporal a través de la estricta postura 
sentada. Dogen lo llama shikan-taza ${ }^{8}$, que equivale a decir que se trata de "sólo sentarse sin más". La meditación en postura sentada intenta volver a experimentar el momento del logro de la iluminación de Buda en el propio cuerpo-mente (DOKYU, 2006). Se tiene que subrayar que la sabiduría búdica no es entendimiento intelectual, sino que se contempla directamente lo natural de la espiritualidad humana a través de la propia interioridad del sí mismo: el Zen es la experiencia introspectiva del propio sí mismo, el camino para acercarse al corazón de Buda y actualizar la naturaleza búdica (la realidad tal cual) dentro de uno mismo (YUASA, 1992, p. 26).

De la misma manera, el pensador Y. Yuasa desarrolla el pensamiento de T. Watsuji sobre el ser humano que existe en el espacio, en relación, y en la naturaleza, y dice que existir en la espacialidad significa para el ser humano la realidad del propio cuerpo que dice "yo estoy aquí", algo similar a la experiencia del satori budista. En efecto, la existencia de la corporalidad en el espacio, según la observación de Yuasa, es una clave definitiva para la comprensión del ser humano de Watsuji.

En la tradición del pensamiento oriental se acentúa la corporalidad, sin que por ello se reduzca a las solas funciones fisiológicas en detrimento de la conciencia humana y del espíritu, por el contrario se insiste en la inseparabilidad de todas las cosas sean tenidas en su subjetividad y objetividad, en su concepto de cuerpo y espíritu o mente, y se subraya la existencia en la unidad de cuerpo-espíritu-naturaleza.

La palabra "corporalidad" se escribe en japonés como Shin-tai, vocablo compuesto por dos caracteres chinos que significan respectivamente "el ser propio y el cuerpo", por tanto se puede traducir como la "corporalidad subjetiva”. Es decir, una manera de contemplar la corporalidad como el sí mismo: no existe otro yo que el cuerpo: un cuerpo que dice "yo".

8 En efecto, la palabra Shikantaza significa literalmente estar simplemente sentado, lo que indica que mientras se medita en zazen no se piensa en ningún objeto, pensamiento o imagen. Se concentra la atención y la actividad principalmente en la espiración, de manera que tiende a establecerse un ritmo lento de respiración abdominal en que la espiración es notoriamente más prolongada que la inspiración. Simplemente estar sentado; no reflexionar; sencillamente respirar y no seguir activamente los pensamientos. No forzar los pensamientos, permitir que fluyan, no detenerse en ellos, dejar que aparezcan y se vayan. Los pensamientos van y vienen, depurando el contenido superfluo del inconsciente, hasta que la mente, entra en un estado de atención más sutil. 
Aquí surge la idea sobre la inseparabilidad de la corporalidad con relación a la mente o el espíritu.

Conviene subrayar que la subjetividad a la que se alude en la tradición japonesa, se refiere a una subjetividad dentro de una actitud de receptividad; es decir, no se trata de un sujeto que se afirma fuertemente a sí mismo y, cuando se abre a lo transcendente, lo hace transcendiendo hacia fuera o hacia arriba, sino que, como afirma el pensador Yuasa, transcenderá hacia la interioridad, buscando el auténtico sentido de la experiencia humana a través de la vida cotidiana en el auténtico "Yo" oculto en lo hondo de la conciencia (YUASA, 1992, p. 10).

Esta noción coincide con la tradición del budismo zen, que alude hacia un transcender hacia dentro. "Hacia dentro" se refiere a un doble sentido: hacia dentro del cuerpo, arraigando la conciencia en la corporalidad, y hacia el fondo del inconsciente: "transcender trans-descendiendo". En las tradiciones de la filosofía occidental, cuando se habla de la corporalidad, hay una tendencia a partir del pensamiento dualista, observando el cuerpo desde una perspectiva exterior, la del cuerpo como objetivado. Después se relaciona y se habla de unidad entre ambos, pero desde un punto de partida que los había separado. Por el contrario, las tradiciones orientales han partido a menudo de vivencias originarias de unidad. Según estas tradiciones del pensamiento oriental, el ser humano existe en la totalidad de la vivencia natural: en el mismo budismo zen se dice (shuzyô) que significa que lo esencial de la vida humana es convivir con todas las cosas que tienen vida, incluidos los animales y las plantas.

En este marco, donde la naturaleza no es considerada como objeto a captar, tampoco existe una manera de observar la corporalidad como un objeto separado del alma o espíritu. Es decir, existe una convivencia del cuerpo y del espíritu desde el principio. La palabra (shin-shin), escrita con caracteres chino-japoneses, está justamente compuesta de dos ideogramas, el de cuerpo y el de espíritu; antes de relacionar los dos, ya proporciona la visión de su unidad.

De la misma manera el pensador Yuasa observa que en las tradiciones del pensamiento oriental no se conoce la separación entre cuerpo y espíritu. Eso es porque en estos ámbitos se aborda la corporalidad desde la perspectiva de la corporalidad subjetiva. Yuasa tiene en cuenta dos 
motivos de la existencia de esa diferencia: en primer lugar, esa diferencia proviene de la manera de contemplar la naturaleza, como se ha visto en el apartado anterior. En segundo lugar, es la cuestión sobre la muerte. En occidente se acentúa más la eternidad de la muerte más allá de la vivencia. Cuando se tiene que afrontar la muerte, no se puede evitar que surja la idea de la separación entre espíritu y cuerpo. Mientras, en Oriente, hay la tendencia de no cuestionar sobre la muerte particularmente. Tanto la vida como la muerte están en la vida eterna universal: unidad entre vida y muerte (seishi-ichinyo), por tanto, dice Yuasa, no hay dualismo de cuerpo y espíritu (YUASA, 1999, p. 63-64).

Es posible que al reflexionar sobre esta concepción de la unidad cuerpo-espíritu, desde la tradición japonesa, se puede aseverar que la tarea más importante para el ámbito del lejano oriente acerca de lo humano es: ¿cómo se puede recuperar, mediante el auto-cultivo, la unidad originaria corpóreo-espiritual de la que nos alejamos en el dualismo de la vida cotidiana?

Es importante desde el budismo zen, fuertemente arraigado en la cultura japonesa, decir que se trata de un auto-cultivo cuyo objetivo es recuperar la unidad corpóreo-espiritual-mental del ser humano. La cultura oriental da mucha importancia a la vinculación del ser humano con la naturaleza ${ }^{9}$. A partir de esa base, se han desarrollado diversas corrientes filosófico-espirituales. En este marco, no se tratan la contemplación y la meditación solamente como un ejercicio espiritual, sino también como ascesis corporal. El carácter peculiar del pensamiento oriental estriba en considerar las prácticas de auto-cultivo o la experiencia como fundamento de la filosofía. Es decir, la sabiduría no se puede conseguir meramente

9 Con respecto a la relación del cuerpo humano con la naturaleza, se puede mencionar el fenómeno de las estaciones, dice así el pensador japonés Toshihiko Izutsu: "La primavera es la época que brotan y florecen el cielo y la tierra, todos los seres, por tanto, hay que levantarse pronto, pasear en el jardín, peinarse bien y relajar el cuerpo intentando despertar la fuerza y ofrecer más que quitar y alabar más que castigar. El verano es la época de crecer, por tanto, hay que dormir pronto, evitar el sol durante el día sin despertar pasión de rabia y intentar no encerrar el 'ki' dentro del cuerpo. En el otoño, el clima es fresco entonces, hay que estabilizar la mente cultivando energía vital y disfrutando de tomar el 'ki' de otoño. En inverno, se esconde la energía positiva, por tanto, hay mantener la mente pasivamente para no sacar energía fuera" (IZUTSU, 1997, p. 34) 
con la reflexión teórica, pero se puede alcanzar a través de la experiencia corporal (taitoku).

Para terminar, Al hablar de la importancia del cuerpo en unión indisoluble con la mente y el espíritu, para entrar en la contemplación, el monje zen Chih-I propone en su obra Pararse a contemplar: hay que ordenar la respiración como método fundamental para cuidar del cuerpo en unión con la mente y la respiración en el cuidado del sí mismo, así lo expresa:

Hay un alto en el camino, detenerse: he aquí el primero y más importante ejercicio práctico necesario para dominar las pasiones: es lo que llamamos pararse, hacer una pausa para respirar; Cuidar el cuerpo, acompasar la respiración y ajustar la mente (CHIH-I, 2007, p. 21)

Esta indisolubilidad entre la mente, el cuerpo y el espíritu, constituye quizás el más importante legado de las tradiciones del lejano Oriente y que contribuye a una mejor comprensión de la condición humana en un mundo cada vez más caótico, paradójico y complejo.

\section{Conclusiones}

Aunque no es tarea fácil conclusiones de este extenso y complejo tema del concepto de cuerpo y corporeidad en el lejano Oriente, se pueden apuntar algunas de las que se consideran primordiales según este estudio:

a) Desde los diversos enfoques del pensamiento del lejano Oriente, se entiende el cuerpo desde su unidad armónica con la mente y el espíritu. Cultivar la unidad del conjunto corpóreo espiritual, es indispensable desde los ejercicios del auto-cultivo como el yoga y el zen. La meditación, la contemplación y los ejercicios físicos, son las maneras de cultivar cuerpo-mente-espíritu y a menudo se han empleado como recursos de prevención y terapia para mantener una buena condición de salud psicobioespiritual. Aquí se incorpora el papel de la respiración que es un componente esencial de los 
ejercicios o entrenamientos de auto-cultivo, como se ha desarrollado en el anterior apartado.

b) Hay que señalar que en el marco del pensamiento del lejano Oriente, una manera de cuidar la vida humana, tanto como condición de la salud física como manera de vivir en el sentido ético, es tratar de vivir bien desde la perspectiva psicosomática, ambiental y comunitaria o social. En otras palabras, la finalidad de cuidar la vida en la tradición del autocultivo no es solamente el bienestar de un individuo, sino que es también el bienestar en relación interpersonal, en la comunidad y de ésta con la misma naturaleza.

c) La iluminación o satori en la tradición del budismo zen es la unión del cuerpo, la mente y el espíritu con la naturaleza, lo que hace tornar a lo natural al ser humano. El mismo ejemplo se puede exponer para mostrar que la finalidad del entrenamiento ( $k e i k o$ ) de las actividades físicas como el yoga, el tai chi o el chi kung, es para alcanzar unidad entre la técnica y el cuerpo-mente. En este marco, el sabio es uno que reconoce esa unidad desde sus experiencias. En la tradición de la cultura oriental es algo natural que un sabio, uno que supera el mundo de la normalidad mediante las prácticas o las formaciones del autocultivo, tenga su bienestar del cuerpo-mente, sea un personaje virtuoso y lleno de longevidad.

d) En el lejano Oriente, se considera que el cuerpo de cada individuo no es su posesión propia, sino que es un don de sus antepasados y la unidad entre tierra y mundo. Esto implica que cuidar el propio cuerpo significa una manera de corresponder a los ancestros por el beneficio de la vida que han otorgado. Se dice en japonés: "El ser humano ha recibido su propio cuerpo de sus padres. La actitud de cuidar de sí mismo para no destruirlo es el primer paso en la búsqueda de la piedad filial". Esa afirmación es un elemento básico del pensamiento sobre la corporalidad del propio sí mismo en Oriente.

e) La importancia que tiene en las tradiciones del lejano Oriente la respiración, hace percibir la relación de convivencia entre la corporalidad y la misma naturaleza. En el marco de la medicina oriental, se considera que el flujo del " $k i$ ", que circula dentro del cuerpo 
humano, fluye hacia fuera a través de los meridianos, sobre todos los puntos de las manos y de los pies llamados seiketsu. Este dato indica que la corporalidad es como un sistema abierto hacia fuera y es inseparable de la naturaleza. Es decir, el pensamiento sobre la salud y la enfermedad oriental está fundado en la visión de la unidad corpóreo-espiritual, así como en la unidad del cuerpo y la naturaleza.

f) Desde las tradiciones del lejano Oriente, se acentúa la espiritualidad dentro de la corporalidad o dentro de la naturaleza. A partir de esa unidad corpóreo espiritual, se aborda la búsqueda de la esencia de lo humano mediante la práctica del autocultivo. Por tanto, la transcendencia tiene dirección hacia adentro del ser humano, no hacia fuera o arriba: la unidad corpóreo espiritual es un camino a la metafísica a través de la metapsicología (YUASA, 2001, p. 299-301).

\section{Referencias}

CHIH-I. Pararse a contemplar: manual de espiritualidad del budismo Tendai. Salamanca: Sígueme, 2007.

DOKYU, R. Introducción a la práctica de Zazen. Buenos Aires: Editorial Kier, 2006.

GARCÍA BACCA, J. D. Elogio de la técnica. Barcelona: Anthropos, 1987.

GOMEZ AREVALO, J. A. La conciencia en el hinduismo: lectura desde la objetividad y subjetividad de la conciencia unificada. Hallazgos: Revista de Investigaciones, n. 6, p. 133-148, 2006.

GUÉNON, R. Introducción General al estudio de las doctrinas hindúes. Barcelona: Editorial Obelisco, 1964.

IZUTSU, T. Sufismo y taoísmo: estudio comparativo de conceptos filosóficos clave. Barcelona: Editorial Siruela, 1997. v. 2. (El Árbol del Paraíso).

LAO-TSÉ. Tao Te Ching: los libros del Tao. Madrid: Trotta, 2006. 
LAO-TSÉ. Camino hacia la virtud [Tao- Te- King]. Tokyo: Editorial Chuokoronsha, 1997.

MASIÁ CLAVEL, J. Buda y los budismos. Madrid: Editorial Santa María, 1996. OSHO. El libro de los chakras. Madrid: Arkano Books, 2012.

YUASA, Y. Ki shugyo Shintai: attunement through the body. New York: Suny, 1992.

YUASA, Y. Shintaino-ucyûsei: Tôyô to Seiyô [La corporalidad cósmica: en Oriente y en Occidente]. Tokio: Editorial Iwanami-shoten, 1999.

YUASA, Y. Shintai-ron: tôyôteki shin-shinron to gendai [El cuerpo: perspectiva oriental sobre cuerpo y mente]. Tokyo: Editorial Kodansha, 2001.

ZUBIRI, X. Sobre el hombre. Madrid: Alianza, 1986.

Recibido: 03/12/2013

Received: 12/03/2013

Aprobado: 26/01/2014 Approved: 01/26/2014 